\title{
From Veneers to Thineers: Two Case Reports with three years follow up.
}

\author{
Dr. Panna Mangat ${ }^{1}$, Dr. Rajesh Podar $^{2}$, Dr. Anjali Miglani ${ }^{3}$ \\ (Conservative Dentistry and Endodontics, DJ College of Dental Sciences and Research, India) ${ }^{1,3}$ \\ (Conservative Dentistry and Endodontics, Terna Dental college, India) ${ }^{2}$
}

\begin{abstract}
This article presents two cases of aesthetic rehabilitation Case 1) Discoloured anterior teeth (tetracycline staining) and Case 2) Diastema closure using minimal intervention dentistry by use of no preparation or minimally preparation teeth. In this Case report porcelain thineers are fabricated which provides best aesthetics with minimal preparation. The key learning points of the article are the following: Proper diagnosis, treatment plan and proper execution of the treatment plan are mandatory for a successful and pleasing result.
\end{abstract}

Keywords: Aesthetic Rehabilitation, Adhesive Technology, Minimal Invasive Dentistry, Thineers, Treatment Planning

\section{Introduction}

While the treatment objectives of today's dentistry regarding the restoration of form and function are unchanged, the demands on quality and esthetics by the patient have grown. ${ }^{1,2}$ Modern material and adhesive techniques have innovated dentistry towards restoration which are truly invisible to the patient. ${ }^{3,4}$ With the improvement of ceramic material and luting composites ceramics and successfully bonded to teeth, conceptualising towards Minimally Invasive Dentistry. ${ }^{5,6}$ Porcelain thinners offer a predictable and successful treatment modality in prosthetic dentistry. The aim of a veneer preparation is to avoid extensive tooth preparation and provide reinforcement of residual tooth structure. Quality of esthetics achieved with bonded restoration is considered a state of Art. Following the facial veneer preparation the increase in flexure can amount to $91 \%$ as measured by 3D FEA. ${ }^{7}$ Bonded ceramic veneers are capable of restorting the fracture strength of teeth to values of intact teeth. ${ }^{4,8,9,10}$ Porcelain veneers are perceived to be one of the most conservative means of restoring aesthetic anterior teeth. Their indications include discoloration, tetracycline staining, flurosis, diastema closure, malformed and malpositioned teeth. ${ }^{11,12,13}$

Purpose of the clinical report is to emphasise on conservative method of aesthetic rehabilitation thus preserving the vitality of the teeth.

\section{Case Report:}

Case I:- A 40yr old male patient reported with chief complaint of discoloured (tetracycline stained) anterior teeth.

Case report 2: A 31 year old patient reported with chief complaint of spacing between upper anterior teeth.

A comprehensive examination was conducted including caries detection, periodontal probing, intraoral and extraoral soft tissue and TMJ examination. Findings were within normal limits and therefore were considered non-contributory. The masticatory and cervical muscles were palpated. Determination was made that occlusion was physiologic and that the patient should be restored to a position of MID.

Following a detailed clinical examination the objective parameters of the patient's smile were carefully evaluated. Intra and extraoral photographs were taken in order to aid in the aesthetic evaluation. Study models were obtained with Reprosil (Dentsply/ Caulk, Milford, DE,USA).

Ceramic thineers were best suited for the condition. These thineers have the advantage of preserving most of the natural tooth structure while achieving all the cosmetic aids. These can be prepared as thin as $0.3 \mathrm{~mm}$. Prior to beginning preparation of the teeth, the color of ceramic thineers was chosen and incisal guidance was checked. All the essential information required to successfully complete the restorative care was gained at the preliminary stage, prior to fabrication of the definitive restoration.

Diagnostic casts were mounted in an articulator, and a preliminary diagnostic wax-up was done. The potential aesthetic benefits of the recommended treatment were demonstrated to the patient using a putty stent obtained from the wax-up of the proposed treatment. 


\subsection{Preparation}

Case 1- Only a minor reduction of the incisal one third $(1 \mathrm{~mm})$ of the lower anteriors was done to create an incisal stop.

Case 2- No preparation.

Polyvinyl siloxane final impressions (Imprint II, 3M Dental Products) were made of the prepared teeth, and master casts were fabricated. The master casts were cross-mounted with the casts of the wax-up using centric relation interocclusal records permitting efficient attainment of correct tooth length, positioning of the midline, duplication of crown contours, and orientation of the occlusal plane. Fit, natural appearance, translucency and the absence of the black triangle in the gingival area were checked.

All of these parameters had been previously determined in the wax up and had been accepted by both the patient and the dentist. The definitive restorations were fabricated and cemented.

\subsection{Thineer manufacture:} Vivadent).

According to manufacturer's instructions, IPS- E max thineers were fabricated. (IPS Emax, Ivoclar

\subsection{Luting procedure:}

Rubber dam and retraction cord was placed to prevent gingival fluids from contaminating the teeth during bonding process.

Before bonding, both surfaces had to be conditioned. Conditioning of ceramic consisted of degreasing with acetone, etching with Hydrofluoric acid (Vita ceramic etch, Vita Zahnfabrik; Bad Sackingen,Germany) for 30seconds, rinsing for 60 seconds and applying Silane (Monobond-S Ivoclar Vivadent) for 60 seconds.

Teeth were conditioned according to the following protocol. After cleaning and polishing with brushes and polishing paste, the enamel was etched with $37 \%$ phosphoric acid (Total Etch, Ivoclar Vivadent) 30 seconds and for dentin 15 seconds.

After thoroughly rinsing and drying the dentin was rewetted with a moist Appli tip (SDI Cari-Tip, Vasbi, Sweden). Syntac primer (Ivoclar Vivadent ) and adhesive was applied for 15 seconds and dried with a gentle blast of air followed by Syntac adhesive (Ivoclar-vivadent) according to manufacturer's instructions. After evaporation of the solvent, Heliobond Resin(Ivoclar-vivadent) was applied in thin layer.

The thineers were bonded with high viscosity dual polymerizing resin composite (Variolink II, Base: Catalyst, translucent shade, Ivoclar Vivadent). Base and catalyst were mixed in a ratio 1:1 ratio and applied to inner surface of the restoration. The thineers were luted using an ultrasonic device (Master Piezon 400, EMS;Nyon, Switzerland) with a modified rubber tip (SP-Tip, EMS). After seating the veneers with luting cement on the preparation excess luting resin was removed with a brush and before light curing the resin composite the veneer margin was covered with glycerine gel (Liquid Strip; Ivoclar Vivadent) to prevent oxygen inhibition layer. Luting interface was light polymerized (Spectrum 800, Dentsply De Trey; Konstanz, Germany) from facial, palatal, mesial and distal interproximal aspects for 60 seconds each, resulting in total polymerization time of 240 seconds. After setting, floss was gently placed into the interproximal areas to remove uncured resin. The margins were finished with finishing diamonds (Gebr. Brasseler) and aluminium oxide polishing disks (Sof Lex 3M; Broken, Germany).

Patient was recalled for routine check up every six months over a period of three years for marginal integrity, aesthetic, oral hygiene maintenance, durability of the restoration, for any visible cracks, debonding, discoloration, gingival inflammation. At the end of three years follow up the clinical outcome was aesthetically pleasing and immaculate.

Case 1- Teteracycline Staining

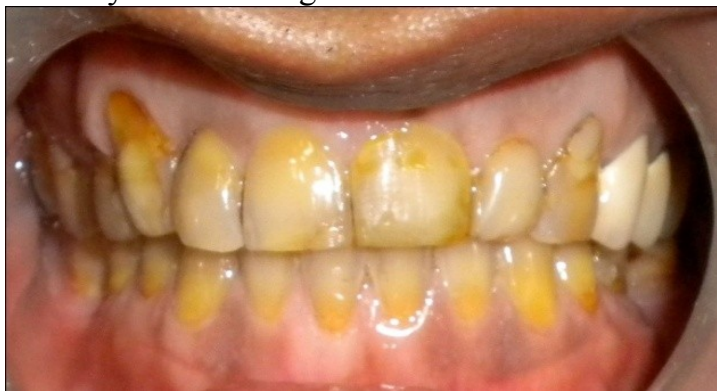

Pre-operative

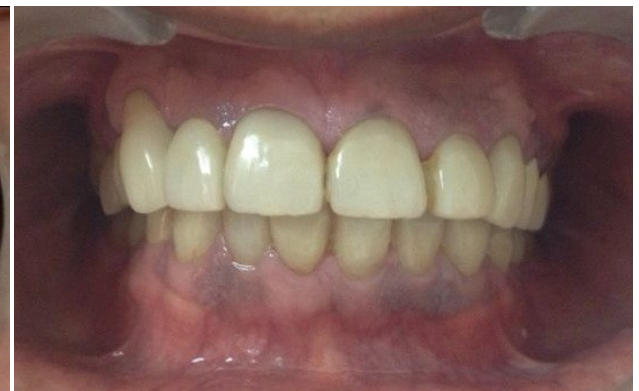

Post-operative 


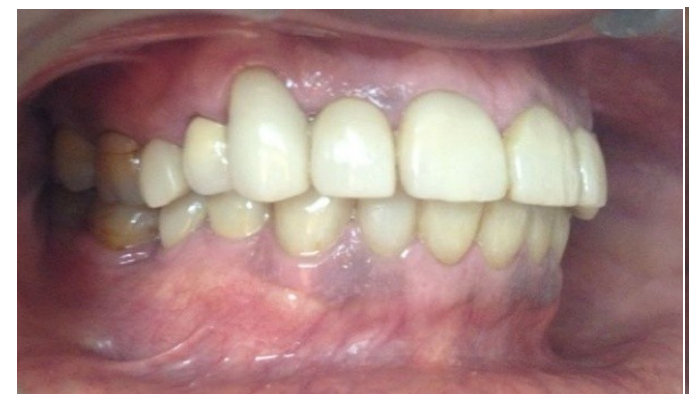

Lateral View right

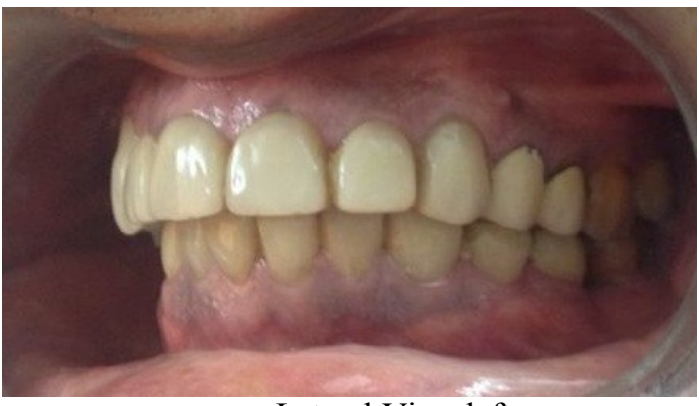

Lateral View left

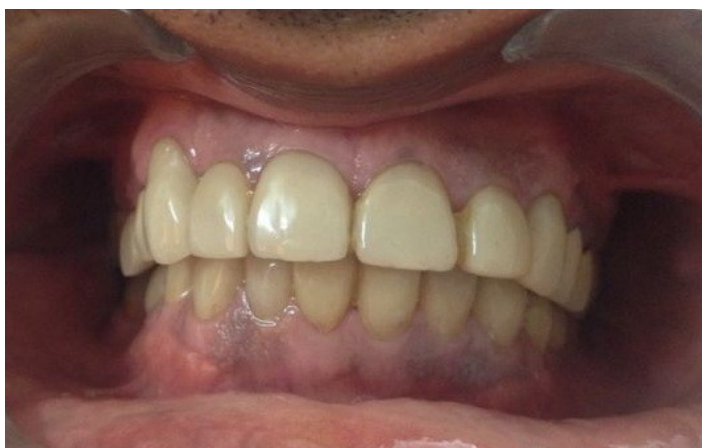

Post-Operative Three Years

Case 2 - Diastema Closure

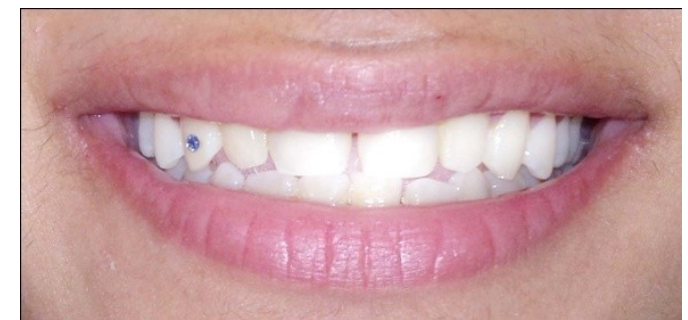

Pre-operative

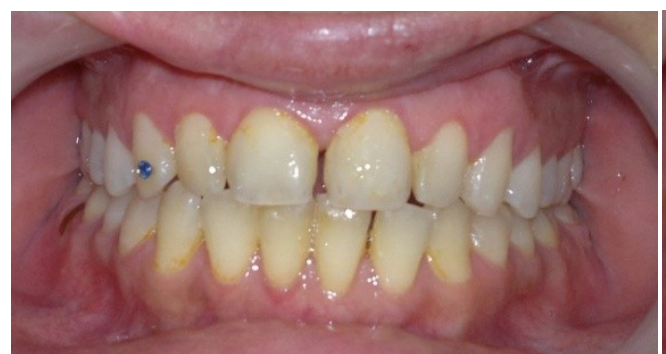

Pre-operative Intraoral

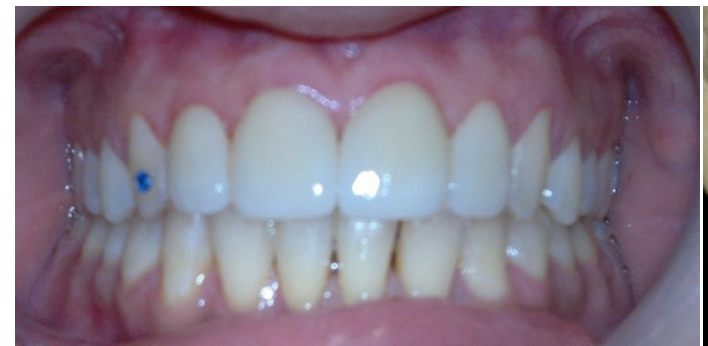

Post Operative Three years

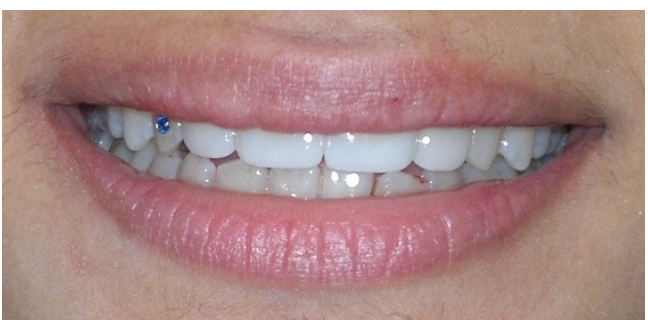

Post-operative

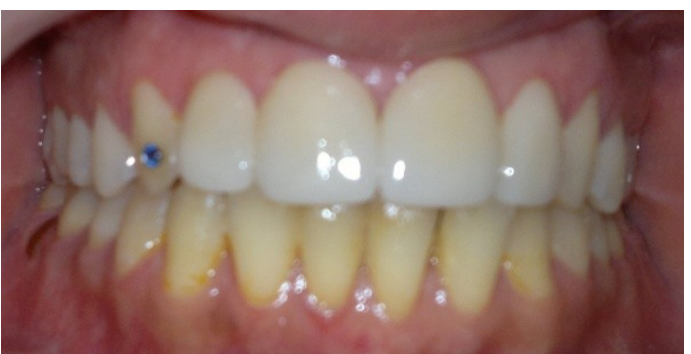

Post-operative Intraoral

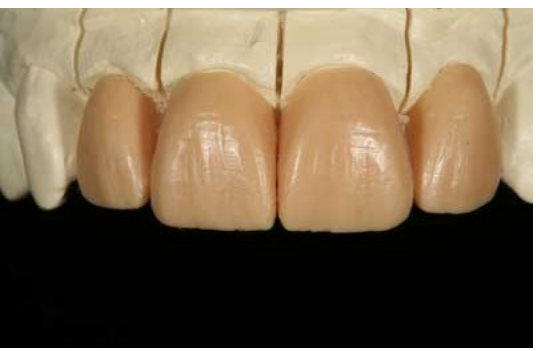

Wax Up 


\section{Discussion}

It is the responsibility of the clinician to fully understand the limitations of the veneers in masking the severe discolorations, taking in tot account that each Ceramic system is unique to its optical properties.

This will ultimately affect the final esthetics of the restoration, the type of ceramic needs to be chosen according to each clinical situation.

Bonded ceramic veneers have a number of significant advantages over metal ceramic / all ceramic crowns. One of the most important advantage is that they are extremely conservative in terms of tooth structure. The most common complications for crown in general are loss of pulp vitality and fracture of the core. Comparing the volume of tooth structure removed for an all-ceramic crown preparation to a veneer preparation, double the amount of tooth structure is removed for a crown than a veneer. ${ }^{14}$

Conservation of the tooth structure is a major factor in determining the long term prognosis of any restorative procedure. Another advantage of the ceramic veneers is their durability. This durability allows minimal reduction resulting in reduced potential pulpal involvement. The periodontal response is beautiful. The restoration can blend imperceptibly with the cervical tooth structure, allowing the cervical margins to be kept in supragingival position. ${ }^{15}$

Theses ceramic veneers should be placed in enamel however with contemporary dentin bonding systems margins can be successfully placed on the dentin/cementum when necessary.

The combination of hydrofluoric acid etching with a silane coupling agent seems to be superior for conditioning the intaglio surfaces of the glass ceramic laminate veneers in comparison to other methods. ${ }^{16,17,18}$

It was even demonstrated that adhesion of resin cements on hydrofluoric acid etched and silanized ceramic had higher bond strength than the same luting cement bonded to enamel. ${ }^{19}$ This combination is stable after long term water strength and thermocycling. ${ }^{16,17,18}$ Process of silanization promotes the wettability on the ceramic and composite surfaces and reacts with the silica surfaces to form covalent bonds. ${ }^{16}$

The success of treatment with ceramic veneers can be assured if the dentist follows a defined protocol with each patient to ensure that all factors such as smile design, margin placement, material and shade selection are considered.

\section{Conclusion}

Aesthetic assessment prior to initiating treatment is critical to achieving the best outcome. Proper guidance in protrusive and lateral excursions must be established to ensure longevity of the restoration. Selection of the best materials to achieve these parameter is of utmost importance. A systematic approach leads to more predictable outcome.

\section{References}

[1]. Heinlein WD. Anterior teeth: esthetics and function. Journal of Prosthetic Dentistry 44, 1980,389-393.

[2]. Mc Lean JW. Aesthetics in restorative dentistry: the challenge for the future. British Dental Joural 149, 1980,368-374.

[3]. Magne P, Douglas WH. Rationalization of esthetic restorative dentistry based on biomimetics. Journal of Esthetic Dentistry 11, $1999,5-15$

[4]. Magne P, Besler U. Bonded porcelain restorations in the anterior dentition: A biometric approach. Chicago: Quintessenc 2002.

[5]. Simonsen RJ. The preventive resin restoration: a minimally invasive, non metallic restoration. Compendium 8,1987, 428-432.

[6]. Tyas MJ, Anusavice KJ, Frencken JE, Mount GJ. Minimal intervention dentistry, a review. FDI Commision Project 1-97. International Dental Journal 50, 2000, 1-12.

[7]. Magne P, Tan DT. Incisor compliance following operative procedure: A rapid 3-D finite element analysis using micro CT data. Journal of Adhesive Dentistry 10, 2008, 49-56.

[8]. Andreasen FM, Flugge E, Daugaard Jensen, Munskgaard EC. Treatment of crown fractured incisors with laminate veneer restorations. An experimental study. Endod Dental Traumatology; 8, 1992, 20-35.

[9]. Magne P, Douglas WH. Design optimization and evolution of bonded ceramics for the anterior dentition. A finite element analysis. Quintessence International. 30, 1999, 661-672.

[10]. Stappert CF, Ozden U, Gerds T, Sturb J.R.Longevity and failure load of ceramic veneers with different preparation designs after exposure to masticatory simulation. Journal of Prosthetic Dentistry 94, 2005, 132-139.

[11]. Weinstein A esthetic appliance of restorative materials and techniques in the anterior dentition. Dental Clinics of North America 37(3), 1993,391-409.

[12]. Lim C. Case selection for porcelain veneers. Quintessence International 26(5),1995, 311-315.

[13]. Peumans M, Van Meerbeek B, Lambrechts P, Vanherle G. Porcelain veneers: A review of the literature. Journal of Dentistry 28(3), 2000, 163-177.

[14]. Edelhoff D, Sorensen JA. Tooth structure removal associated with various preparation designs for anterior teeth. Journal of Prosthetic Dentistry 87, 2002,503-509.

[15]. Shailendra Gupta, Deepak Raisingani, Pradyumna Misra. Esthetic rehabilitation with ceramic veneers: A Case Report. International Journal of Contemporary Dentistry; 2(1), 2011, 13-16.

[16]. Blatz MB, Sadan A, Kern M. Resin- ceramic bonding: a review of the literature. Journal of Prosthetic Dentistry 3, $2003,268-274$.

[17]. Bltaz MB, Sadan A, Maltezos C, BLatz U, Mercante D, Burgess JO. In vitro durability of the resin bond to feldspathic ceramics. American Journal of Dentistry 17, 2004,169-172.

[18]. Brentel AS, Ozcan M, Valandro LF, Alarca LG, Amarai R, Bottino MA Microtensile bond strength of a resin cement to feldspatic ceramic after different etching and silanization regimens in dry and aged conditions. Dental Materials 23, 2007, 1323-1331.

[19]. Penumans M, Meerbeek van B, Lambrechts P, Vanherle G. Porcelain veneers: a review of the literature. Journal of Dentistry 28, 2000, 163177. 